\title{
Evaluación biofísica de servicios ecosistémicos en la cuenca del Arroyo Grande, Tunuyán, Mendoza
}

\author{
Jorgelina Teruya ${ }^{1,}{ }^{\otimes} ;$ Leandro Mastrantonio $^{1}$ \& José Portela ${ }^{2}$ \\ ${ }^{1}$ Facultad de Ciencias Agrarias, Universidad Nacional de Cuyo, Argentina. ${ }^{2}$ Estación Experimental Agropecuaria INTA La \\ Consulta.
}

\begin{abstract}
Resumen. La cuenca del Arroyo Grande (Valle de Uco, Tunuyán, Mendoza) es un área que presenta cambios clave en el uso de sus recursos, producidos principalmente por los nuevos emprendimientos vitivinícolas y facilitados por la aplicación del riego presurizado. En estos cambios primó la decisión privada y la diversidad de criterios de gestión ambiental. Con el objetivo de evaluar la capacidad funcional de los dos ecosistemas principales, el monte nativo y el agroecosistema, y la provisión relativa de servicios ecosistémicos (SE) en la cuenca del Arroyo Grande, se aplicó una metodología basada en el protocolo ECOSER 1.0 mediante un sistema de información geográfica. A partir de información biofísica del área se ubicaron en el espacio los tipos de ecosistemas dominantes (TED) y se consideraron cuatro funciones ecosistémicas (FE). La identificación de cada SE y su provisión relativa se calculó como la combinación lineal del flujo de cada FE ponderado por su aporte relativo al correspondiente SE. Por último, la provisión de SE se evaluó espacialmente mediante la generación de mapas. Los resultados muestran al agroecosistema como proveedor de un mayor número de SE, dentro de los ocho analizados. Entre ellos figura la producción agrícola, que incluye cultivos, ganado y forestación. El monte nativo provee en mayor proporción SE de soporte y aprovisionamiento, relacionados con la provisión de agua y su calidad, que a su vez son esenciales para el agroecosistema. En conclusión, existen diferencias en la provisión de SE según los ecosistemas, y aquellos que brinda el agroecosistema dependen de otros SE, proporcionados antes por el monte nativo.
\end{abstract}

[Palabras clave: funciones ecosistémicas, variabilidad espacial, frontera agrícola]

Aвstract. Biophysic assessment of ecosystem services in the Arroyo Grande basin, Tunuyán, Mendoza. The Arroyo Grande basin (Valle de Uco, Tunuyán, Mendoza) is an area that shows major changes in the use of natural resources, primarily generated by new investments for wine-grape production, and facilitated by the application of pressurized irrigation. Private decisions, as well as a diversity of criteria for environmental management, prevailed in those changes. In order to evaluate the functional capacity of the two major ecosystems identified, shrubland and agroecosystem, and their relative provision of ecosystem services (ES) in the Arroyo Grande basin, we applied a methodology based on the ECOSER 1.0 protocol, using a geographic information system. Using biophysical information from the area, we identified and mapped the dominant ecosystems types (TDE). We considered four ecosystem functions (EF) and eight ES. The identification of each ES and its relative provision was calculated as a linear combination of the flow of each EF weighted by their relative contribution to the corresponding ES. Finally, through the generation of maps, we evaluated spatially ES provision. Results show the agroecosystem provides a larger number of ES, including agricultural production as crops, livestock and forestry. The shrubland provides a greater proportion of supporting and provisioning ES, related to water supply and quality, which, in turn, are essential for the agroecosystem. In conclusion, there are differences in the provision of ES according to the ecosystems, and those ES provided by the agroecosystem depend on another ES previously provided by the shrubland.

[Keywords: ecosystem functions, spatial variability, agricultural frontier]

\section{INTRODUCCIÓN}

En los últimos años, en el Valle de Uco (Mendoza, Argentina) se han producido reestructuraciones y cambios clave en el modo técnico y social de producción de los sistemas agroalimentarios. Esto tuvo consecuencias para su ruralidad ya que cambió la realidad en la que deben actuar los sistemas de extensión y transferencia de tecnología (Pizzolato et al. 2010), como así también los de gestión y administración del territorio. En la zona, tradicionalmente de

Editor asociado: Matías Mastrangelo

jorgelina.teruya@gmail.com producción vitivinícola bajo el sistema de riego por canales, se produjo un avance rápido de la frontera agrícola sobre el piedemonte, en un proceso caracterizado por nuevos grandes emprendimientos, facilitado por la aplicación de sistemas de riego presurizado en base al uso masivo de agua subterránea, orientado a implantar uvas finas para vinificar (Pizzolato et al. 2010). En este cambio en el uso del suelo primaron la decisión privada y el empleo de criterios completamente diversos respecto de la gestión que debía hacerse del ambiente transformado y de su entorno. 
El estudio de los servicios ecosistémicos (SE) establece un vínculo explícito entre el bienestar humano y el funcionamiento adecuado del ecosistema (Balvanera et al. 2011). Cualquier decisión de manejo puede tener efectos negativos o positivos sobre los diferentes SE y conducir a relaciones de compromiso entre ellos. El principal desafío es definir cómo decidir la asignación y la administración óptima de múltiples opciones de uso de la tierra (De Groot et al. 2009), a fin de generar enfoques, modelos y marcos conceptuales que se sustenten en los diagnósticos de nuestra realidad para conformar soluciones propias, novedosas y funcionales. Para ello, existen herramientas de evaluación y mapeo de SE, robustas en cuanto a su integración de elementos y lo suficientemente amigables como para facilitar su adopción, que permiten responder a demandas concretas (Nahuelhual et al. 2013). Entre ellas se encuentra el protocolo ECOSER 1.0 para el mapeo de servicios ecosistémicos (Laterra et al. 2011). El objetivo de este trabajo fue evaluar la capacidad funcional y la provisión relativa de SE de los principales ecosistemas presentes en la cuenca del Arroyo Grande, ubicada en el Valle de Uco.

\section{Materiales y Métodos}

\section{Área de estudio}

El estudio se realizó en la cuenca media y baja del Arroyo Grande, entre los $33^{\circ} 34^{\prime}$ y los $34^{\circ} 37^{\prime} \mathrm{S}$ y los $69^{\circ} 00^{\prime}$ y $69^{\circ} 23^{\prime} \mathrm{O}$. Esta zona corresponde políticamente al departamento de Tunuyán, que integra la región denominada Valle de Uco, en la Provincia de Mendoza (Argentina). El clima es árido, con temperatura media anual de $15{ }^{\circ} \mathrm{C}$ y un régimen de precipitación estival, con un promedio anual de $320 \mathrm{~mm}$. El esquema hidrogeológico de la cuenca es sencillo, ya que la misma se adosa a la Cordillera Frontal mediante una bajada pedemontana que culmina en una llanura aluvial de poco desarrollo, con una pendiente marcada hacia el noreste. Constituye una depresión rellenada con sedimentos aluviales entre el terciario y el cuaternario. El proceso de colmatación de la depresión, provocado por el depósito de los materiales acarreados por los ríos y arroyos, se produjo a través de una sedimentación selectiva, lo que dejó los clastos más gruesos cerca de la cordillera y los más finos en la parte distal, al este-noreste. Esto, además de la topografía, determina la presencia de una zona de recarga y otra de descarga de los acuíferos. Los suelos son de escaso desarrollo, clasificados como Entisoles. La flora es característica de la provincia fitogeografía del Monte. La fisonomía del valle se completa con amplias áreas cultivadas, principalmente con frutales (Martinis et al. 2002). En el contexto social, encontramos que la mayoría de los habitantes son asalariados agrícolas e industriales, dueños de empresas, empleados públicos y comerciantes (Pizzolato and Costella 2012).

\section{Compilación de datos}

Se compiló información de textura de suelos, coberturas del terreno y modelo digital de elevaciones empleando una imagen LANDSAT del 27/02/2011. El NDVI se calculó mediante el programa QGIS 2.6.1 Brighton, y se identificaron y localizaron espacialmente los tipos de ecosistemas dominantes (TED).

\section{Análisis de datos}

Se utilizó el protocolo ECOSER 1.0 como base de cálculo de las funciones y servicios ecosistémicos (Laterra et al. 2011). Se consideraron y mapearon cuatro funciones ecosistémicas (FE): almacenamiento de carbono en biomasa, almacenamiento de carbono orgánico en el suelo, control de la erosión por cobertura vegetal y protección de acuíferos mediante coberturas vegetales. El cálculo del almacenamiento de carbono en la biomasa consistió en asignar un factor por defecto de contenido de carbono en biomasa a cada uso/cobertura. Los factores empleados se extrajeron de bibliografía. En el caso de la cobertura agroecosistema, el factor, con un valor de $5.67 \mathrm{t} \mathrm{C} /$ ha, se tomó de datos globales propuestos por el protocolo ECOSER 1.0. En el caso del monte nativo se aplicó el valor 3.8 t C/ha (Zivkovic et al. 2013).

La función almacenamiento de carbono orgánico en el suelo se obtuvo a partir de la metodología propuesta por el IPCC (Intergovernmental Panel on Climate Change) (IPCC 2006). Esta metodología lo estima como el producto del valor de carbono orgánico del suelo bajo las condiciones de referencia $\left(\mathrm{COS}_{\text {Ref }}\right)$ (vegetación nativa sin disturbar), expresado en $t$ /ha $y$ obtenido de muestras de suelo a través del análisis del porcentaje de materia orgánica (MO) oxidable, con el Método de Walkley-Black (1934), multiplicado por tres factores de cambio que se indican en la Ecuación 1, extraídos de bases bibliográficas del IPCC (2006). Estos factores $\left(\mathrm{F}_{\mathrm{lu}^{\prime}}, \mathrm{F}_{\mathrm{mg}}, \mathrm{F}_{\mathrm{a}}\right)$ se refieren a cambios relacionados con el tipo de 
cobertura del suelo, a las diferentes prácticas de laboreo del suelo realizadas y a los distintos niveles de aporte de carbono al suelo (Tabla 1).

$$
\operatorname{CoS}_{i}=\operatorname{CoS}_{R e f} \times F_{l u} \times F_{m Q} \times F_{a} \quad(\text { Ecuación 1) }
$$

Para el caso del control de la erosión, estimamos esta función utilizando la ecuación universal de pérdida de suelo revisada (RUSLE) (Renard et al. 1997), que predice pérdidas de sedimentos en medias anuales de acuerdo al índice de erosividad por precipitaciones $(\mathrm{R})$, al factor de erodabilidad de suelos (K), la longitud (L) y a pendiente del terreno(S), al factor de cobertura (C) y al factor de prácticas de conservación $(\mathrm{P})$. Calculando la diferencia entre las tasas de erosión sin cobertura vegetal en una situación hipotética de suelo desnudo (Ecuación 2) y las tasas de erosión con la cobertura existente (Ecuación 3), estimamos cuál es el control que realiza la vegetación sobre la pérdida de sedimentos (Ecuación 4).

$$
\begin{aligned}
& A_{\text {máx }}=R \times K \times L S \\
& A_{i}=R \times K \times L S \times C \times P \\
& A_{\text {máx }}-A_{j}
\end{aligned}
$$

(Ecuación 2)

(Ecuación 3)

(Ecuación 4)

El factor R se define como el producto acumulado de la energía cinética de una precipitación por su máxima intensidad en 30 minutos, para un período de interés con cierta probabilidad de ocurrencia (50\%). En este estudio, debido a que no se disponía de la información específica requerida, se empleó el valor $\mathrm{R}$ anual calculado para la región pedemontana del Gran Mendoza (Vich et al. 2010). Los datos estadísticos de precipitación mensual se extrajeron de la Dirección de Agricultura y Contingencia Climática, de la estación meteorológica Tunuyán, correspondientes al período 1997-2011.

El factor Kes una medida dela susceptibilidad de las partículas del suelo para separarse y ser transportadas por la lluvia y la escorrentía. Se obtuvo aplicando la ecuación propuesta por Wischmeier y Smith (1978).

$$
Y=-0.402 \times X+48.928 \quad \mathrm{R}^{2}=0.39 \text { (Ecuación 6) }
$$

donde $\mathrm{Y}$ es el porcentaje de arena muy fina (50-100 micrómetros) y X es el valor de arena total (50-2000 micrómetros).

El porcentaje de materia orgánica $(\mathrm{MO})$ se obtuvo a partir de las muestras de suelo, que se analizaron mediante el análisis de \%MO oxidable con el método de Walkley-Black (1934). Para el código de estructura (E) se estableció un valor de 4 (Wischmeier and Smith 1978) ya que los suelos de la zona poseen una estructura en bloques o carecen de estructura. El código de permeabilidad (P) se estableció por la clase de conductividad hidráulica saturada, a partir de los datos de contenido de arcilla y arena (USDA 1993). LS indica el producto de los sub-factores longitud (L) y pendiente (S) del terreno, y representa el efecto de la topografía sobre la pérdida de sedimentos por erosión. El método aplicado coincide con el expuesto en el protocolo ECOSER (Wischmeier and Smith 1978). El factor L se calculó con la expresión:

$$
L=\left(\frac{X}{22} \times 13\right)^{m}
$$

(Ecuación 7)

donde $X$ representa la longitud de la pendiente en porcentaje, que es la distancia horizontal, pendiente abajo, desde el punto donde se genera el escurrimiento hasta el punto donde la pendiente decrece de forma que comienza la deposición de sedimentos. La longitud se derivó a partir del MDE. Para el cálculo del factor $\mathrm{S}$ se empleó la Ecuación 8, en la que 's' es la pendiente en porcentaje.

$$
s=\left(0.065+0.045 \times s+0.0065 \times s^{2}\right) \quad(\text { Ecuación } 8)
$$

Tabla 1. Factores relativos de cambio de existencias de carbono en el suelo.

Table 1. Related factors of soil carbon stock change.

\begin{tabular}{lccc}
\hline TED & $\mathrm{F}_{\mathrm{lu}}$ & $\mathrm{F}_{\mathrm{mg}}$ & $\mathrm{F}_{\mathrm{a}}$ \\
\hline Agroecosistema & 0.8 & 1.01 & 1.04 \\
Monte nativo & 1 & 1.10 & 1
\end{tabular}

$\mathrm{F}_{\mathrm{lu}}: \overline{\text { factor de cambio relacionado con el tipo de cobertura/ }}$ uso del suelo; $\mathrm{F}_{\mathrm{mg}}$ : factor de cambio relacionado con las diferentes prácticas de labores; $\mathrm{F}_{\mathrm{a}}$ : factor de cambio que representa los distintos niveles de aporte de carbono al suelo.

$$
1.292 \times \frac{\left[2.1 \times M 1.14 \times 10^{-4} \times(12-M O)+3.25 \times(E-2)+2.5 \times(P-3)\right]}{100}
$$

(Ecuación 5)

donde $\mathrm{M}=(100-\%$ arcilla $)$ * (\% limo+\% arena muy fina); $\mathrm{MO}=\%$ materia orgánica; $\mathrm{E}=$ código (curva) de estructura y $\mathrm{P}=$ código (curva) de permeabilidad. Los datos empleados para el cálculo del término $\mathrm{M}$ de la ecuación fueron obtenidos a partir del análisis de laboratorio de 23 muestras de suelo del área de estudio (Mastrantonio et al. 2012). El porcentaje de arena muy fina se obtuvo por medio de la siguiente regresión: 
En consecuencia, el factor topográfico LS se calculó a partir de:

$L S=\left(\frac{X}{22} \times 13\right)^{m} \times\left(0.065+0.045 \times s+0.0065 \times s^{2}\right)$
(Ecuación 9)

donde LS representa la proporción de pérdida de suelo en comparación con un sitio de referencia "estándar", con 9\% de pendiente y $22.13 \mathrm{~m}$ de longitud de pendiente (Wischmeier and Smith 1978).

Los factores C y P representan la reducción de las pérdidas de sedimentos por protección de cobertura vegetal y buenas prácticas de manejo o conservación, respectivamente. Para la zona en estudio, P es igual a la unidad ya que en el monte nativo no existe ninguna labor cultural que pueda atenuar la erosión, y en el agroecosistema, por el tipo de cultivo (e.g., vid, frutales, etc.), las labores son mínimas. Para el valor $\mathrm{C}$ se generó un mapa raster empleando la herramienta Factor C de Qgis, basado en el NDVI (Ecuación 10):

$$
C=e^{-\alpha \frac{N D V I}{\beta-N D V I}}
$$

(Ecuación 10)

donde: $\alpha=2$ y $\beta=1$.

Por último, para estimar la protección de acuíferos mediante las coberturas vegetales se asumió que cada tipo de cobertura presenta una determinada protección del acuífero frente a la contaminación, en función del nivel de agroquímicos que se requieren para cada actividad (estimado a través de un factor de ponderación). Esta protección es más relevante donde el acuífero es más vulnerable. El protocolo plantea la siguiente ecuación para su cálculo:

$$
P A C_{i}=1-\left(I D_{\text {reli }} \times P_{i}\right)
$$

(Ecuación 11)

donde $\mathrm{PAC}_{\mathrm{i}}$ es la protección de acuíferos por cobertura en el pixel i, $P_{i}$ es un factor que refleja el rol de protección de la cobertura vegetal en el pixel i en función del nivel de insumos necesarios para el desarrollo de la actividad productiva. ID reli $_{\text {es el índice DRASTIC }}$ relativizado, empleado como medida de la vulnerabilidad del acuífero en el pixel i.

El método DRASTIC (Aller et al. 1987) clasifica y pondera parámetros intrínsecos, reflejo de las condiciones naturales del medio como: D (profundidad del nivel piezométrico), $\mathrm{R}$ (recarga), A (litología del acuífero), S (naturaleza del suelo), T (pendiente del terreno), I (naturaleza de la zona no saturada) y C (conductividad hidráulica del acuífero - permeabilidad) (Ecuación 12).
A cada uno de los siete parámetros se les asigna un valor en función de los diferentes clases y rangos definidos (Tabla 2). Además, al valor de cada variable se le aplicó un índice de ponderación entre 1-5 que cuantifica su importancia relativa y puede modificarse en función del contaminante.

Para asignar el factor Pi se consideró una modificación de los valores que se encuentran en el protocolo ECOSER 1.0 (Laterra et al. 2011). Los cultivos en el protocolo poseen un valor de $\mathrm{Pi}=95 \% \mathrm{y}$, suponiendo que el uso de agroquímicos es menor en el caso analizado por los tipos de cultivos presentes en la zona de estudio, se eligió un valor de Pi de $70 \%$ en el caso del agroecosistema y se empleó un valor $\mathrm{Pi}=5 \%$ para el monte nativo, considerando la presencia de escasa ganadería extensiva.

La identificación de cada SE y su provisión relativa se obtuvo a partir de la integración de los mapas de FE. Para ello, las funciones fueron normalizadas según los valores máximo y mínimo debido a que los mapas de funciones tienen unidades diferentes. Se generó, además, una matriz de valoración del aporte relativo (bj) de cada FE a cada SE a través de consultas a expertos $(n=11)$ provenientes del medio académico (ingenieros agrónomos) y de gestión (organismos técnicos) que trabajan en la temática y/o en el área de estudio. Estos fueron previamente informados sobre la problemática planteada, el objetivo del proyecto y sobre las definiciones de los conceptos básicos de la metodología a aplicar. El valor del aporte relativo se estableció en un rango de 0 a 1 , donde 0 correspondía a un aporte bajo o despreciable y 1 a las funciones que ejercían la máxima contribución dentro de cada servicio. Se consideraron ocho SE relevantes para la zona (Tabla 4 ).

La provisión se calculó como la combinación lineal del flujo de cada función ecosistémica (FEj) ponderado por su aporte relativo (bj) al correspondiente SE (Ecuación 13).

$$
F S E_{i}=\sum b_{j} \times F E_{j}
$$

(Ecuación 13)

Por último, mediante la generación de mapas se evaluó espacialmente la provisión de SE listados en la Tabla 4, elegidos en función del protocolo.

$$
I D=D_{i} \times D_{w}+R_{i} \times R_{w}+A_{i} \times A_{w}+S_{i} \times S_{w}+T_{i} \times T_{w}+I_{i} \times I_{w}+C_{i} \times C_{w}
$$

donde i es el pixel evaluado y w el índice de ponderación de cada parámetro (Tabla N³). 
Tabla 2. Rango de valores de los parámetros empleados en el índice DRASTIC.

Table 2. Range of values of the parameters used in the DRASTIC index.

\begin{tabular}{|c|c|c|c|c|c|c|c|c|c|c|c|c|c|}
\hline \multicolumn{2}{|c|}{$\mathrm{D}(\mathrm{mm})$} & \multicolumn{2}{|l|}{$\bar{R}(\mathrm{~mm})$} & \multicolumn{2}{|l|}{$\bar{A}$} & \multicolumn{2}{|l|}{$\mathrm{S}$} & \multicolumn{2}{|c|}{$\mathrm{T}(\%)$} & \multicolumn{2}{|l|}{$\mathrm{I}$} & \multicolumn{2}{|c|}{$\mathrm{C}$ (m/día) } \\
\hline$<1.5-10$ & 8.5 & $0-50.8$ & 1 & & & Arenoso & 9 & $0-2$ & 10 & Arena, & 6 & Acuifero & \\
\hline $10-20$ & 5 & 50.8-101.6 & 3 & & & $\begin{array}{l}\text { Franco- } \\
\text { arenoso }\end{array}$ & 6 & $2-6$ & 9 & $\begin{array}{c}\text { grava y } \\
\text { limo }\end{array}$ & & $\begin{array}{c}\text { confinado } \\
42-82\end{array}$ & 8 \\
\hline $20-30$ & 2 & $101.6-117.8$ & 6 & $\begin{array}{c}\text { Arena y } \\
\text { grava }\end{array}$ & 8 & Franco & 5 & $6-12$ & 5 & $\begin{array}{c}\text { Arena y } \\
\text { grava }\end{array}$ & 8 & Acuifero & 10 \\
\hline$>30$ & 1 & & & & & $\begin{array}{l}\text { Franco- } \\
\text { limoso }\end{array}$ & 4 & $\begin{array}{c}12-18 \\
>18\end{array}$ & 3 & basalto & 9 & lil & 10 \\
\hline
\end{tabular}

Tabla 3. Índices de ponderación empleados en el índice DRASTIC (Aller et al. 1987).

Table 3. Indexes of weighting employees in the DRASTIC index (Aller et al. 1987).

\begin{tabular}{lc}
\hline \multicolumn{1}{c}{ Parámetro } & $\begin{array}{c}\text { Índice de } \\
\text { ponderación }(w)\end{array}$ \\
\hline D - Profundidad del acuífero & 5 \\
R - Recarga & 4 \\
A - Naturaleza del acuífero & 3 \\
S - Naturaleza del suelo & 2 \\
T - Pendiente del terreno & 1 \\
I - Impacto de la zona no saturada & 5 \\
C - Conductividad hidráulica & 3 \\
\hline
\end{tabular}

\section{Resultados y Discusión}

Se diferenciaron dos tipos de ecosistemas dominantes (Figura 1). El primero es el agroecosistema, caracterizado por un suelo de textura franco-limosa, con más de $1 \%$ de materia orgánica debido a su agregado por labores culturales en función del requerimiento de los cultivos comerciales que forman la cobertura. El segundo es el monte nativo, con suelo de textura arenosa y franco-arenosa, con menos de $1 \%$ de materia orgánica y sin agregado de la misma por trabajos culturales, con una cobertura vegetal típica de la provincia fitogeografía del Monte.
Tabla 4. Valoración del aporte relativo de cada función (columnas) a cada servicio (filas). 0 : aporte bajo o despreciable; 1 : máxima contribución.

Table 4. Assessment of the relative contribution of each function (columns) to each service (rows). 0 : low or negligible contribution; 1: maximum contribution.

Funciones ecosistémicas

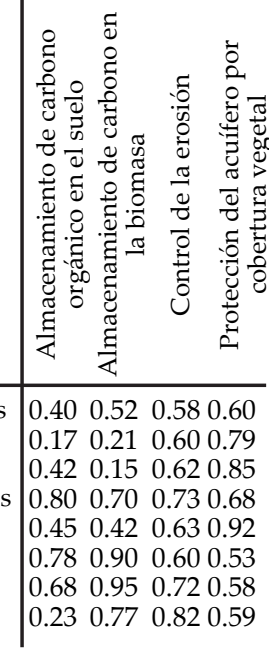

Al analizar los mapas de las FE se observó que el agroecosistema almacena más carbono en la biomasa por el mayor crecimiento, volumen y superficie de dosel que presentan las especies cultivadas bajo riego y, por lo tanto, al sufrir un déficit hídrico menor. Para el caso de almacenamiento de carbono orgánico en el suelo, encontramos áreas del agroecosistema en el extremo inferior de la cuenca con valores

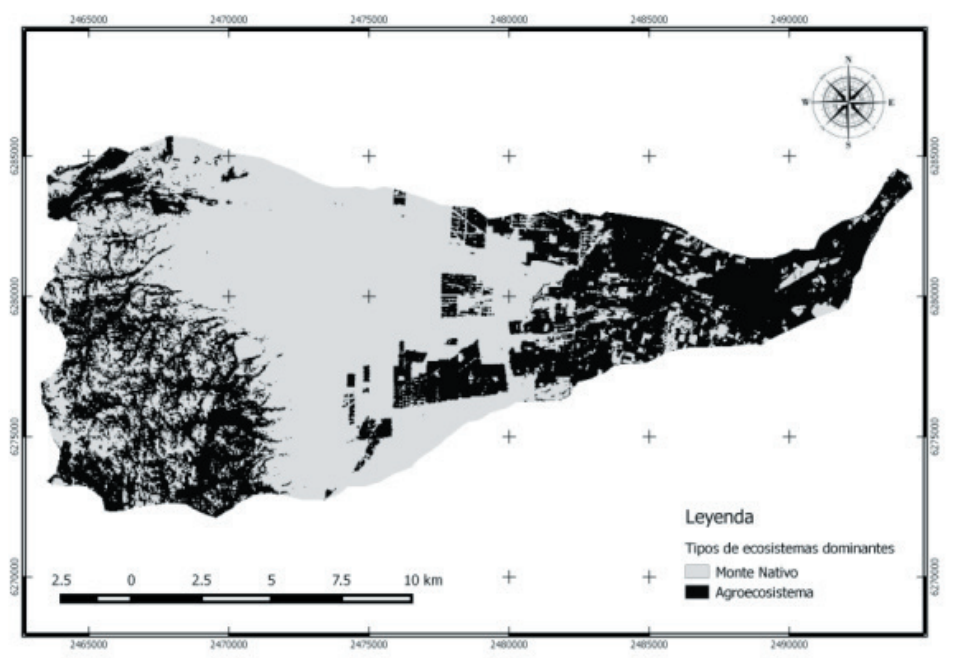

Figura 1. Tipos de ecosistemas dominantes. Agroecosistema y monte nativo.

Figure 1. Types of dominant ecosystems. Agroecosystem and shrubland. 
de almacenamiento mayores a 0.5 (t/ha). Estos valores se explican por los valores altos de COS de referencia en comparación con otras áreas, lo que obedece a la incorporación de material vegetal que realizan los agricultores desde hace años. Esto no ocurre en el monte nativo. Algo distinto ocurre en la parte media de la cuenca, donde los dos TED poseen valores similares entre sí dada la corta historia de la zona en cuanto a prácticas culturales de incorporación de $\mathrm{MO}$, y a que predomina la vid conducida en espaldero y regada mediante goteo. Esto ocasiona una falta de cobertura en el área interfilar. En consecuencia, en este caso, los factores de cambio $\left(\mathrm{F}_{\mathrm{lu}}, \mathrm{F}_{\mathrm{mg}}\right.$ y $\left.\mathrm{F}_{\mathrm{a}}\right)$ no ejercen demasiada influencia sobre el valor final de la función de almacenamiento de carbono orgánico en el suelo.

Respecto al control de la erosión por cobertura vegetal, se concluyó que en la cuenca analizada, el factor de erosión dominante es la pendiente. Por el contrario, la cobertura vegetal ejerce poca influencia sobre el control de la misma ya que no se observan diferencias de este valor, a pesar de que el TED agroecosistema posee mayor cobertura que el TED monte nativo.

En el mapa resultante de protección de acuíferos por cobertura vegetal se destaca una zona con altos valores de protección, que oscilan entre 0.9 y 1 , en correspondencia con el límite del área cultivada y la expansión de la frontera agrícola. El TED agroecosistema es el de menor protección del acuífero debido a su alta vulnerabilidad a la contaminación, definida por el modelo DRASTIC. Esto se debe principalmente a que el acuífero en esa área posee muy poca profundidad, incluso con zonas de surgencia. En el área correspondiente al TED monte nativo aumenta

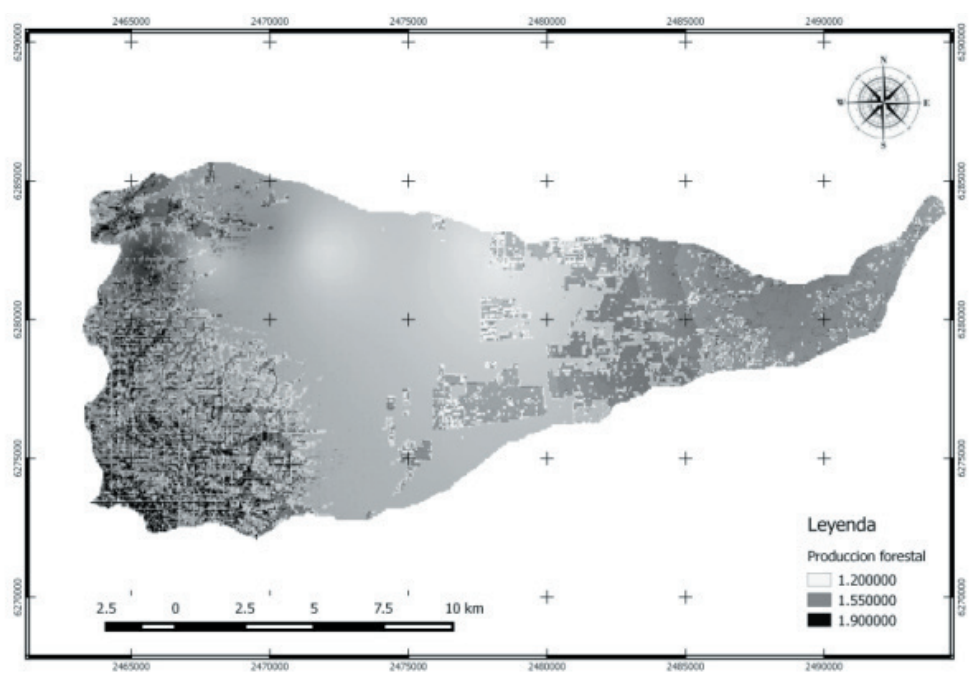

Figura 2. Producción forestal.

Figura 2. Forestry production.

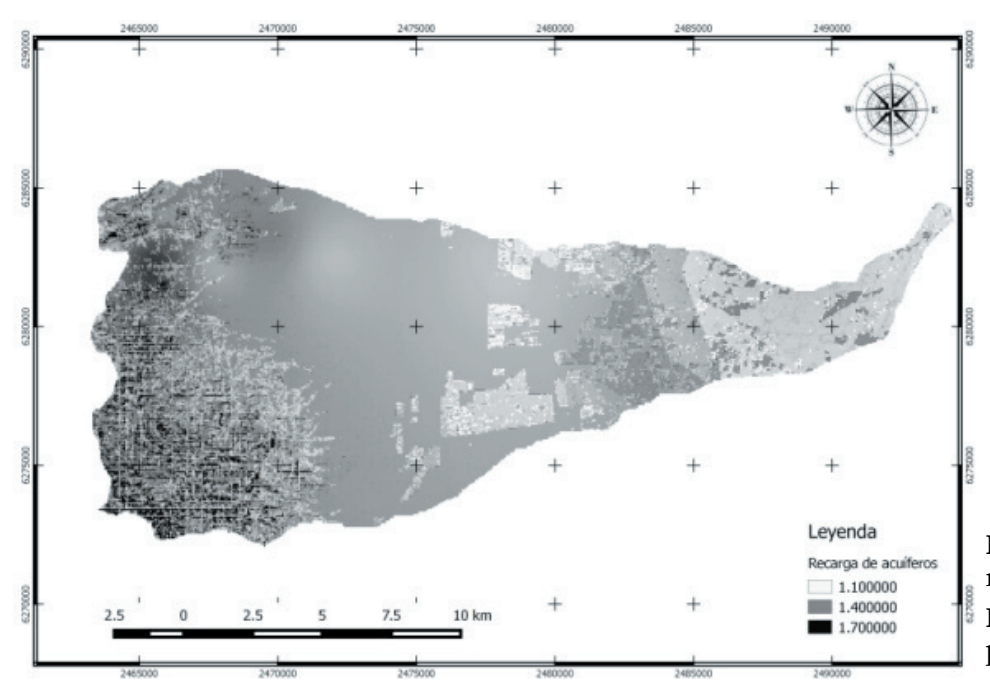

Figura 3. Provisión del servicio recarga de acuíferos.

Figure 3. Aquifer recharges service provision. 
la vulnerabilidad en los límites con las áreas cultivadas. La causa de esto es la existencia de zonas aisladas de cultivo, que en ocasiones están desmontadas y sin cultivar. Del análisis de esta función se concluyó que el factor que más diferencia de vulnerabilidad produce entre los TED es la profundidad del acuífero (factor D en la Ecuación 12).

Respecto a los SE evaluados, la producción forestal (Figura 2), producción ganadera, producción potencial de cultivos y recarga de acuíferos (Figura 3) fueron los de mayor provisión relativa. Las producciones forestal, ganadera y potencial de cultivos presentaron valores de provisión mayores en el agroecosistema que en el monte nativo, sobre todo por la influencia de las FE de almacenamiento de carbono orgánico en el suelo y de carbono en biomasa, que obtuvieron mayor ponderación por parte de los expertos consultados. ElSE recarga de acuífero, presentó dos zonas bien diferenciadas: una de mayor recarga (correspondiente al monte nativo) y otra de menor recarga (en el agroecosistema). Esto puede explicarse debido a la textura más gruesa del suelo del monte, que permite una mejor percolación, a la conductividad hidráulica del suelo y a la existencia de acuífero libre, características derivadas del proceso de formación de este suelo aluvial. En este caso, el SE se genera principalmente en el monte nativo para ser captado como beneficio a través del uso del agua subterránea por los cultivos desarrollados en el agroecosistema. Por lo tanto, seria, conveniente conservar el monte como zona de recarga para mantener el balance hídrico de agua subterránea. Al igual que la recarga de acuífero, el servicio de recarga de lagos y embalses (Figura 4) es

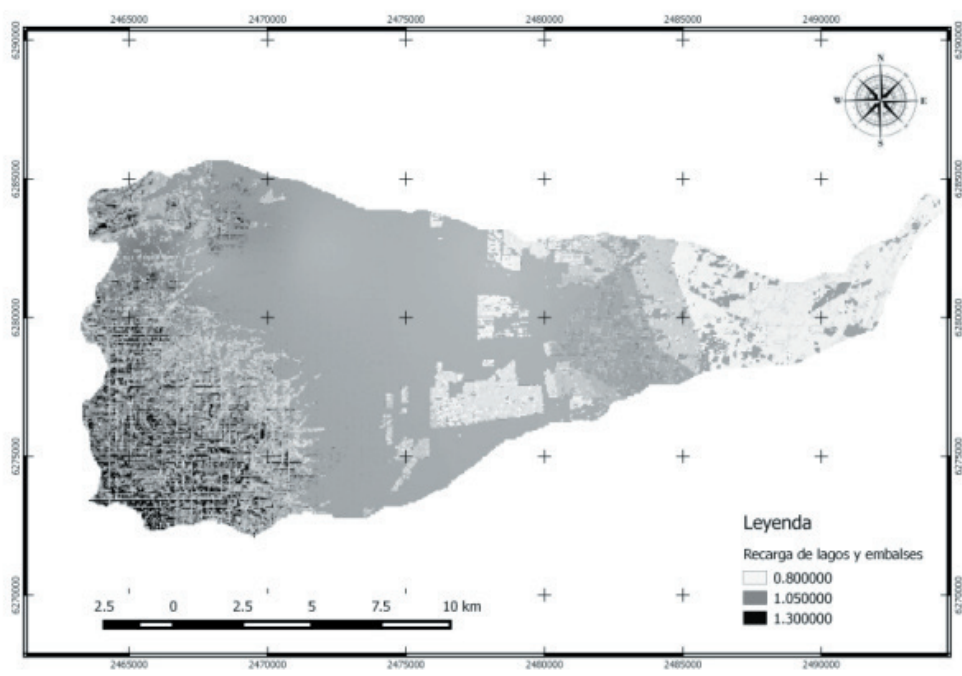

Figure 4. Provisión del servicio recarga de lagos y embalses.

Figure 4. Provision of the service recharge of lakes and reservoirs.

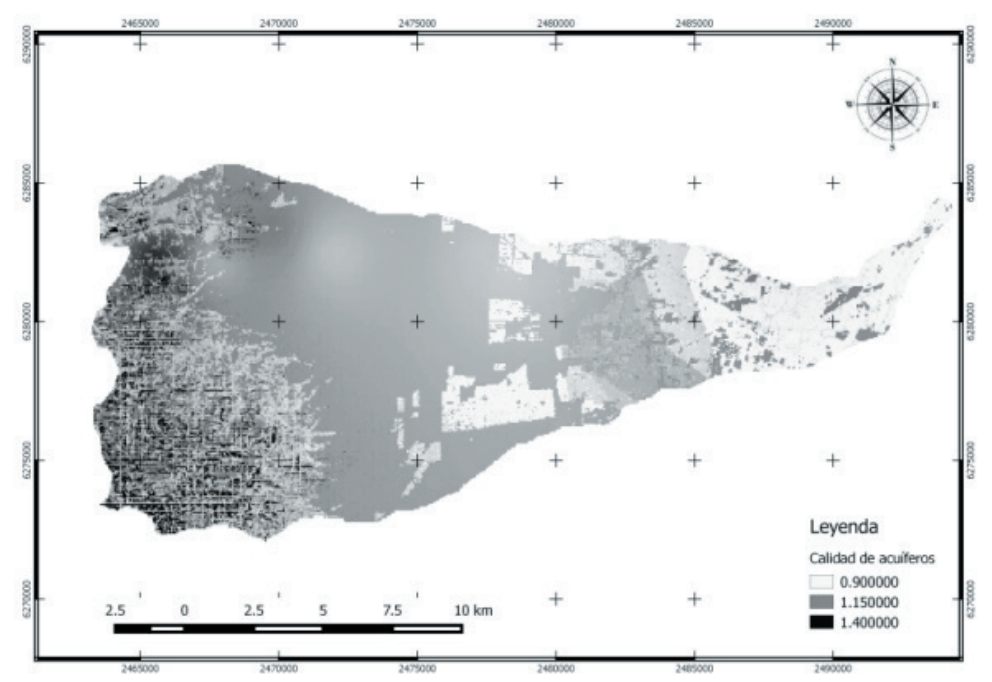

Figura 5. Provisión del servicio amortiguación de inundaciones.

Figure 5. Provision of service buffer of floods. 
provisto relativamente en mayor proporción por el monte nativo, con la salvedad de que la provisión de este servicio es menor en toda la cuenca en comparación con la de recarga de acuíferos.

Los modelos sobre cambio climático para la región cordillerana predicen que las temperaturas altas favorecen las precipitaciones en forma de lluvia, o que la nieve, en sitios altos protegidos, se funda rápidamente. Los cambios ocurridos y los proyectados producirán potencialmente que el hidrograma de los ríos que se originan en los Andes se modifique de manera sustancial, en especial durante la estación seca (Boninsegna et al. 2013). La importancia de considerar estos cambios radica en los posibles impactos sobre los SE que presta nuestra Cordillera de los Andes, como son la recarga de acuíferos que estamos analizando, y otros como la recarga de lagos y embalses.

En el caso del SE de amortiguación de inundaciones (Figura 5), la provisión del servicio es mayor en el agroecosistema, ya que el control de la erosión hídrica es más alto debido a la mayor densidad de plantas, a la mejor estructura del suelo cultivado por las prácticas agrícolas de incorporación de $\mathrm{MO}$ y a la menor pendiente que existe en la zona baja de la cuenca (entre 1\% y $3 \%$ ). No obstante, en general, no se puede aseverar que existan grandes diferencias con el monte nativo.

El monte nativo provee en mayor proporción sólo tres SE, clasificados como de soporte y aprovisionamiento, relacionados con la provisión de agua (Figuras 3 y 4) y su calidad
(Figura 6). El SE de calidad del acuífero en el monte podría explicarse por oposición a las prácticas agrícolas que fomentan la utilización de agroquímicos, que a pesar de no añadirse en grandes cantidades por el tipo de requerimiento de los cultivos de la zona es muy diferente a la ausencia de incorporación, como ocurre en el Monte. El SE de calidad de acuífero, al igual que el de recarga, se genera en la zona del monte nativo para ser percibido en la zona baja de la cuenca, donde se practica la agricultura. Por esto, como se dijo antes, sería conveniente conservar el monte para mantener la calidad hídrica del recurso destinada a sus distintos usos.

El servicio de potencial recreativo presenta valores mayores en el límite de la frontera agrícola y es provisto en gran medida por el agroecosistema. Weyland y Laterra (2013) obtuvieron resultados similares, lo cual confirma que el oasis por regadío es una de las áreas de mayor potencial recreativo, constituyéndose como atractivos de la zona la agricultura vinculada al oasis y la industria asociada a las bodegas.

\section{CONCLUSIONES}

La cuenca del Arroyo Grande (Tunuyán, Mendoza) es un área sujeta a conflictos relacionados con procesos de expansión agrícola que afectan el uso y conservación de sus recursos naturales. Este es un problema que se extiende al resto de la región del Valle de Uco, caracterizada por una complejidad de actores territoriales, producto de las relaciones sociales profundamente desiguales

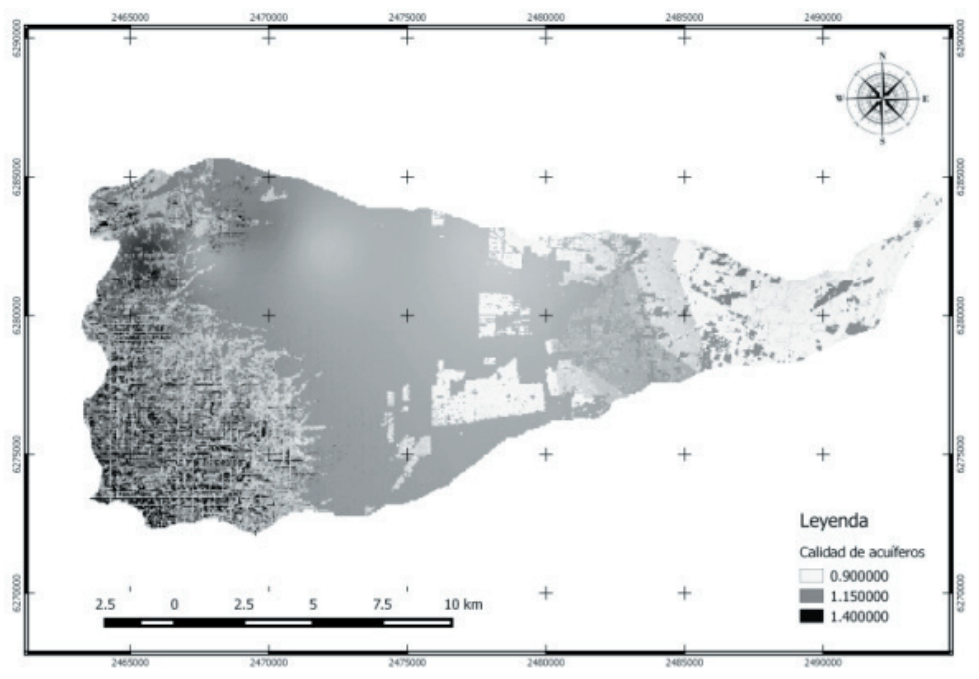

Figura 6. Provisión del servicio calidad de acuíferos.

Figure 6. Provision of the service quality of aquifers. 
y la creciente concentración económica de la producción.

Este trabajo, planteado como estudio exploratorio de aplicación del protocolo ECOSER 1.0 para evaluar la capacidad funcional de ecosistemas y la provisión relativa de servicios ecosistémicos en la cuenca del Arroyo Grande, permite concluir que existen diferencias en la provisión de SE según los usos del suelo. El agroecosistema es proveedor de un mayor número de servicios, dentro de los 8 analizados. Sin embargo, parte de los SE brindados dependen de otros servicios proporcionados por el monte nativo. La función del monte nativo como proveedor de servicios de provisión de agua y de calidad de agua, particularmente en la parte alta de la cuenca, es importante y necesaria en la producción de otros servicios, como los relacionados con el aprovisionamiento de madera, ganado y alimentos, que son brindados por el agroecosistema. Tener en cuenta esta dinámica de la cuenca, plasmada en los mapas de provisión, permitiría tomar decisiones sobre el uso del agua y del suelo que optimicen la oferta conjunta de servicios ecosistémicos y la distribución de los beneficios a ellos asociados.

El presente trabajo constituye un primer antecedente de aplicación del protocolo ECOSER en zonas áridas. En tal sentido, este estudio contribuye al enriquecimiento del protocolo, con la incorporación en la RUSLE del factor de erodabilidad de la precipitación anual $(\mathrm{R})$ desarrollado para la región pedemontana del Gran Mendoza (Vichy Mariani 2010). También amplía el conocimiento existente sobre el monte nativo desde el punto de vista de su importancia en la provisión de servicios ecosistémicos y, más importante aun, aporta la aplicación de una herramienta que puede ayudar en la resolución de problemas productivos y ambientales, y apoyar la toma de decisiones para el ordenamiento territorial, para el uso y manejo de ecosistemas y para la formación de paisajes.

Agradecimientos. A la Secretaría de Ciencia, Técnica y Posgrado de la UNCuyo por la financiación del Proyecto 06/A596, y al INTA por la financiación del PRET MZASJ1251205.

\section{REFERENCIAS}

Aller, L., T. Bennett, J. H. Lehr, R. J. Pertty, and G. Hackett. 1987. DRASTIC: A standardized system for evaluating ground water pollution potential using hydrogeologic settings. NWWA/EPA Series, EPA-600/2-87-035.

Balvanera, P., A. Castillo, E. Lazos Chavero, K. Caballero, S. Quijas, A. Florea, et al. 2011. Marcos Conceptuales interdisciplinarios para el estudio de los servicios ecosistémicos en América Latina. Capítulo 2. Pp. 41-67 en P. Laterra, E. G. Jobbágy y J. M. Paruelo. Valoración de servicios ecosistémicos: conceptos, herramientas y aplicaciones para el ordenamiento territorial. Ediciones INTA, Buenos Aires, Argentina.

Boninsegna, J. A., R. Villalba, and R. Urrutia. 2013. El cambio climático y los servicios del agua en la Cordillera de los Andes. Pp. 263-280 en A. Lara, P. Laterra, G. Barrantes (eds.). Servicios ecosistémicos hídricos: estudios de caso en América Latina y el Caribe. Valdivia, Chile. Red ProAgua CYTED.

De Groot, R. S., R. Alkemade, L. Braat, L. Hein, and L. Willemen. 2009. Challenges in integrating the concept of ecosystem services and values in landscape planning, management and decision making. Ecological Complexity 7:260-272.

IPCC (Intergovernmental Panel on Climate Change). 2006. Capítulo 5, cuadro 5.5. Pp. 20. Inventarios nacionales de gases de efecto invernadero. Vol. 4: Agricultura, silvicultura y otros usos de la tierra. Disponible en: www.ipccnggip.iges.or.jp/public/2006gl/spanish/vol4.html.

Laterra, P., F. Castellarini, and E. Orúe. 2011. Ecoser: Un protocolo para la evaluación biofísica de servicios ecosistémicos y la integración con su valor social. Capítulo 16. Pp. 359-390 en P. Laterra, E. G. Jobbágy y J. M. Paruelo. Valoración de servicios ecosistémicos: conceptos, herramientas y aplicaciones para el ordenamiento territorial. Ediciones INTA, Buenos Aires, Argentina.

Martinis, N., A. Álvarez, J. Hernández, and J. Villalba. 2002. Evaluación hidrogeológica de la cuenca del Valle de Uco. Tomo 1, 2 y 3. Publicación Interna IT No 15-CRA. Instituto Nacional del Agua. Centro Regional Andino. Pp. 520.

Mastrantonio, L., B. Pérez Valenzuela, J. Maffei, M. Pérez, and S. Dágata. 2012. Evaluación de funciones de pedotransferencia en suelos del Valle de Uco, Mendoza. XIX Congreso Latinoamericano y XXIII Congreso Argentino de la Ciencia del Suelo.

Nahuelhual, L., P. Laterra, A. Carmona, N. Burgos, A. Jaramillo, P. Barral, et al. 2013. Evaluación y Mapeo de servicios ecosistémicos: una revisión y análisis de enfoques metodológicos. Pp. 21-38 en A. Lara, P. Laterra, G. Barrantes (eds.). Servicios ecosistémicos hídricos: estudios de caso en América Latina y el Caribe. Valdivia, Chile. Red ProAgua CYTED.

Pizzolato, D., and L. Costella. 2012. Propuesta metodológica para la construcción de grupos sociales en estudios sociales agrarios. El caso del distrito de Vista Flores, Tunuyán, Mendoza. Agencia de Extensión Rural INTA La Consulta.

Pizzolato, D., and P. Potaschner. 2010. Reestructuración de los sistemas agroalimentarios y diferenciación social agraria en el Valle de Uco, Mendoza. VIII Congreso Latinoamericano de Sociología Rural, Porto de Galinhas. 
Renard, K. G., G. R. Foster, G. A. Weesies, D. K Mccool, and D. C. Yoder (coordinators). 1997. Predicting Soil Erosion by Water: A guide to Conservation Planning with the Revised Universal Soil Loss Equation (RUSLE). U.S Department of Agriculture, Agriculture Handbook No 703. Pp. 404.

USDA. 1993. Soil survey manual handbook 18. Soil Survey Division Staff. Soil Conservation Service. Valdez, C., and A. Ruiz Luna. 2011. Marco Conceptual y Clasificación de los Servicios Ecosistémicos. Revista Bio Ciencias 1(4):9.

Vich, A., and A. Mariani. 2010. Evaluación y predicción de la erosión hídrica en regiones áridas de relieve acentuado del centro-oeste de Argentina. Multequina 19(1).

Walkley, A., and I. A. Black.1934. An examination of the Degtjareff method for determining organic carbon in soils: Effect of variations in digestion conditions and of inorganic soil constituents. SoilScience 63:251-263.

Weyland, F., and P. Laterra. 2013. Una propuesta metodológica para el estudio del potencial recreativo a escala de paisaje. Pp. 249-262 en A. Lara, P. Laterra, G. Barrantes (eds.). Servicios ecosistémicos hídricos: estudios de caso en América Latina y el Caribe. Valdivia, Chile. Red ProAgua CYTED.

Wischmeier, W., and D. Smith. 1978. Predicting rainfall erosion losses: A guide to conservation planning. United States Department of Agriculture, Agriculturehadbook N537. Pp. 58.

Zivkov, L., E. Martínez Carretero, A. Dalmaso, and M. Almirón. 2013. Carbono acumulado en la biomasa vegetal de la reserva de Villavicencio (Mendoza-Argentina). Bol Soc Argent Bot [online] 48(3-4):543-551. 